\title{
MRI-Based Attenuation Correction for PET/MRI Using Multiphase Level-Set Method
}

\author{
Hyun Joon An ${ }^{1,2}$, Seongho Seo ${ }^{1,3}$, Hyejin Kang ${ }^{1}$, Hongyoon Choi ${ }^{1}$, Gi Jeong Cheon ${ }^{1,4}$, Han-Joon Kim ${ }^{5}$, Dong Soo Lee ${ }^{1,4,6}$, \\ In Chan Song ${ }^{7}$, Yu Kyeong Kim ${ }^{1,8}$, and Jae Sung Lee ${ }^{1-4}$ \\ ${ }^{1}$ Department of Nuclear Medicine, Seoul National University College of Medicine, Seoul, Korea; ${ }^{2}$ Department of Biomedical \\ Sciences, Seoul National University College of Medicine, Seoul, Korea; ${ }^{3}$ Department of Brain and Cognitive Sciences, Seoul National \\ University College of Natural Sciences, Seoul, Korea; ${ }^{4}$ Institute of Radiation Medicine, Medical Research Center, Seoul National \\ University College of Medicine, Seoul, Korea; ${ }^{5}$ Department of Neurology, Seoul National University College of Natural Sciences, \\ Seoul, Korea; ${ }^{6}$ Department of Molecular Medicine and Biopharmaceutical Sciences, Graduate School of Convergence Science and \\ Technology, Seoul National University, Suwon, Korea; ${ }^{7}$ Department of Radiology, Seoul National University College of Medicine, \\ Seoul, Korea; and ${ }^{8}$ Department of Nuclear Medicine, Seoul Metropolitan Government Boramae Medical Center, Seoul, Korea
}

Inaccuracy in MR image-based attenuation correction (MR-AC) leads to errors in quantification and the misinterpretation of lesions in brain PET/MRI studies. To resolve this problem, we proposed an improved ultrashort echo time MR-AC method that was based on a multiphase level-set algorithm with main magnetic field $\left(B_{0}\right)$ inhomogeneity correction. We also assessed the feasibility of this levelset-based MR-AC method (MR-AC level $_{1}$ ), compared with CT-AC and MR-AC provided by the manufacturer of the PET/MRI scanner (MR$\left.\mathrm{AC}_{\mathrm{mMR}}\right)$. Methods: Ten healthy volunteers and 20 Parkinson disease patients underwent ${ }^{18} \mathrm{~F}-\mathrm{FDG}$ and ${ }^{18} \mathrm{~F}$-fluorinated- $\mathrm{N}$-3-fluoropropyl-2$\beta$-carboxymethoxy-3- $\beta$-(4-iodophenyl)nortropane ( $\left.{ }^{8} \mathrm{~F}-\mathrm{FP}-\mathrm{CIT}\right) \mathrm{PET}$ scans, respectively, using both PET/MRI and PET/CT scanners. The level-set-based segmentation algorithm automatically delimited air, bone, and soft tissue from the ultrashort echo time MR images. For the comparison, MR-AC maps were coregistered to reference CT. PET sinogram data obtained from PET/CT studies were then reconstructed using the CT-AC, MR-AC ${ }_{m M R}$, and $M R-A C_{\text {level }}$ maps. The accuracies of SUV, SUVr (SUV and its ratio to the cerebellum), and specific-to-nonspecific binding ratios obtained using $M R-\mathrm{AC}_{\text {level }}$ and $M R-A C_{m M R}$ were compared with CT-AC using region-ofinterest- and voxel-based analyses. Results: There was remarkable improvement in the segmentation of air cavities and bones and the quantitative accuracy of PET measurement using the level set. Although the striatal and cerebellar activities in ${ }^{18} \mathrm{~F}-\mathrm{FP}-\mathrm{CIT}$ PET and frontal activity in ${ }^{18} \mathrm{~F}$-FDG PET were significantly underestimated by the $M R-A C_{m M R}$, the $M R-A C_{\text {level }}$ provided PET images almost equivalent to the CT-AC images. PET quantification error was re-

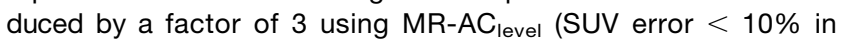

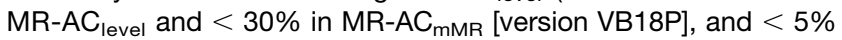
in $\mathrm{MR}^{-} \mathrm{AC}_{\text {level }}$ and $<15 \%$ in MR-AC $\mathrm{MMR}_{\mathrm{m}}$ [VB20P]). Conclusion: The results of this study indicate that our new multiphase level-setbased MR-AC method improves the quantitative accuracy of brain PET in PET/MRI studies.

Received Jul. 9, 2015; revision accepted Nov. 25, 2015.

For correspondence or reprints contact either of the following:

Jae Sung Lee, Department of Nuclear Medicine, Seoul National University

College of Medicine, 103 Daehak-ro, Jongno-gu, Seoul 110-799, Korea.

E-mail: jaes@snu.ac.kr

Yu Kyeong Kim, Department of Nuclear Medicine, Seoul Metropolitan Government Boramae Medical Center, 20 Boramae-ro 5-gil, Dongjak-gu,

Seoul 156-707, South Korea.

E-mail: yk3181@snu.ac.kr

Published online Dec. 23, 2015.

COPYRIGHT (C) 2016 by the Society of Nuclear Medicine and Molecular Imaging, Inc.
Key Words: PET/MRI; attenuation correction; level-set segmentation; brain PET

J Nucl Med 2016; 57:587-593

DOI: $10.2967 /$ jnumed.115.163550

$\mathbf{T}$ he development of tomographic imaging technologies has made dramatic progress in recent decades. Among the modern medical imaging systems, PET and MRI have greatly contributed to understanding normal and abnormal brain functions and evaluating various neurologic disorders (1-4). Although PET is the most sensitive medical imaging device, providing both functional and biochemical information, it has limited spatial resolution, signalto-noise ratio, and anatomic information. Conversely, MRI offers detailed anatomic information about the brain along with excellent soft-tissue contrast and various types of hemodynamic information (i.e., perfusion and diffusion). Accordingly, the combination of PET and MRI can provide a 1-stop shop for clinical examination and new methodology for exploring the brain with multiparametric and complementary imaging information $(5,6)$.

In addition, fully integrated PET/MRI scanners based on semiconductor photosensors, such as avalanche photodiodes and silicon photomultipliers, allow the simultaneous acquisition of both image datasets, which possess several distinct advantages over the sequential scan in conventional PET/CT examinations (7-11). Accurate spatiotemporal correlation of PET/MRI signals permits the studies to demonstrate the relationship between neurotransmitter release and hemodynamic change in the brain under various pathologic and pharmacologic circumstances. Head motion correction of PET images using the motion information derived from the rapidly acquired time series of MR images is another advantage of simultaneous PET/MRI scans. Moreover, the MRIbased extraction of arterial input function for PET kinetic analysis and partial-volume correction of PET has become easier to perform and more accurate $(5,6,9,12-14)$.

However, the accuracy of attenuation correction of brain PET in PET/MRI studies is still questionable. Because the MRI signal is not directly related to the photon attenuation, PET attenuation correction in PET/MRI relies on MRI segmentation, population-based 
standard templates, or joint activity and attenuation estimation (15-18). The template-based method is robust but has limitations in accommodating the wide interindividual anatomic variability in patients' brains (19-22). The MRI segmentation-based method using a 2-point Dixon sequence does not provide the bone segment, leading to the underestimation of uptake around the bone (23-27). Although the reconstruction algorithms for the joint estimation of activity and attenuation have great potential, the PET timing resolution that determines the accuracy of these algorithms is not good enough in current PET/MRI scanners (28-30).

The MRI-based attenuation correction (MR-AC) using an ultrashort echo time (UTE) MRI sequence derives the bone segment based on the difference between 2 MR images obtained at different echo times (ultrashort and typical times) $(31,32)$. Thus, the most widely used clinical PET/MRI system (Biograph mMR; Siemens Healthcare) offers the UTE-based MR-AC along with the Dixon-based method for brain PET/MRI studies. However, the initial versions of the UTE sequence (i.e., mMR software version VB18P) yielded frequent segmentation errors at the boundary between soft tissue, bone, and air, and misclassification of the ventricle as air (33-35). Although a recent upgrade of the software from VB18P to VB20P offers more reliable attenuation maps than before, significant segmentation errors in the regions around the inferior part of the brain (i.e., sinus and lower skull structures) still exist. Moreover, considerable quantification errors because of the inaccurate UTE MR-AC have been reported in several articles (33-35).

Here, we propose an advanced UTE MR-AC method that is based on a multiphase level-set algorithm (36-38) to provide more accurate attenuation maps than those currently used in brain PET/MRI studies. The quantitative accuracy of this new method, providing a 3-segment (air, bone, and soft tissue) attenuation map, was compared with CT-based and mMR-providing attenuation corrections. For this comparison, we used image datasets obtained from ${ }^{18} \mathrm{~F}$-fluorinated- $N$-3-fluoropropyl-2- $\beta$-carboxymethoxy-3- $\beta$-(4-iodophenyl)nortropane $\left({ }^{18} \mathrm{~F}\right.$-FP-CIT) and ${ }^{18} \mathrm{~F}-$ FDG brain PET/MRI and PET/CT studies.

\section{MATERIALS AND METHODS}

\section{Patient Population}

We evaluated our new MR-AC method using 2 different brain PET datasets. One of these was the ${ }^{18} \mathrm{~F}$-FP-CIT PET/CT and PET/MRI data acquired in our previous study for evaluating the accuracy of existing MR-AC methods in patients with Parkinson disease (35). Four more PET studies have been added since the publication of the previous study; thus, a total of 20 patients ( 11 men, 9 women; mean age \pm $\mathrm{SD}, 59.6 \pm 9.1 \mathrm{y}$; age range, 54-71 y) were enrolled in this study. The other was ${ }^{18} \mathrm{~F}$-FDG PET/CT and PET/MRI data of 10 prospectively enrolled healthy normal volunteers ( 6 men, 4 women; mean age $\pm \mathrm{SD}$, $57.7 \pm 5.4 \mathrm{y}$; age range, 51-67 y) without any medical diseases or abnormalities uncovered in neuropsychologic screening tests. All these studies were approved by the Institutional Review Board of our institute, and all study participants signed an informed consent form.

\section{PET/CT and PET/MRI Acquisition}

PET/CT data were acquired using a Biograph mCT40 scanner (Siemens Healthcare) in ${ }^{18} \mathrm{~F}$-FP-CIT studies and a Biograph TruePoint 40 scanner in ${ }^{18} \mathrm{~F}$-FDG studies. A Biograph mMR system was used for PET/MRI data acquisition in both studies. Although the mMR software version of VB18P was used in the ${ }^{18} \mathrm{~F}-\mathrm{FP}$-CIT studies, the software was upgraded to VB20P for the ${ }^{18} \mathrm{~F}-\mathrm{FDG}$ studies. The VB20P is the latest version and provides improved UTE image quality by incorporating gradient delay correction, streak artifact suppression, and a more advanced MR-AC method. In this new version, skull segment is generated using a template-based approach and combined with the softtissue map obtained by applying MRI segmentation.

In the ${ }^{18} \mathrm{~F}$-FP-CIT studies, a PET/MRI scan was obtained $110 \mathrm{~min}$ after the injection of a tracer (192 MBq on average) and followed by a PET/CT scan. In the ${ }^{18} \mathrm{~F}-\mathrm{FDG}$ studies, the sequence of PET/MRI and PET/CT was randomly determined, and the first scan was obtained $40 \mathrm{~min}$ after the injection of the tracer (259 $\mathrm{MBq}$ on average). PET scan duration for ${ }^{18} \mathrm{~F}$-FP-CIT and ${ }^{18} \mathrm{~F}$-FDG was $10 \mathrm{~min}$.

PET/CT Acquisition. PET/CT imaging was performed in a single PET bed position and the participants' heads were positioned in a head holder attached to the patient bed. The PET/CT scan followed the routine clinical protocol for brain studies including a topogram scan, an attenuation CT scan, and a 10-min PET emission scan. For PET attenuation correction, the $\mathrm{CT}$ images were reconstructed in a $512 \times 512 \times 112$ matrix with voxel sizes of $0.59 \times 0.59 \times 3 \mathrm{~mm}$. The emission PET data were acquired in sinogram format.

PET/MR Acquisition. In PET/MRI, the participants' heads were positioned in the mMR head coil. MR images were acquired simultaneously with PET using a dual-echo UTE sequence (echo time, 0.07 and $2.46 \mathrm{~ms}$; repetition time, $11.9 \mathrm{~ms}$; flip angle, $10^{\circ}$ ). The UTE images were reconstructed into a $192 \times 192 \times 192$ matrix with an isotropic voxel size of $1.33 \mathrm{~mm}$ (Supplemental Fig. 1; supplemental materials are available at http://jnm.snmjournals.org). A T1-weighted 3-dimensional ultrafast gradient echo sequence was also acquired in a $208 \times 256 \times 256$ matrix with voxel sizes of $1.0 \times 0.98 \times 0.98 \mathrm{~mm}$.

\section{MR-Based Attenuation Map Using Level-Set Algorithm}

The $\mathrm{T} 2$ relaxation time of protons in bone tissue is much faster than in other tissues. Thus, bone tissues with short $\mathrm{T} 2$ can be distinguished from soft tissue by taking the subtraction or division between the first UTE image (UTE1) and the second longer TE image (UTE2). However, these images, especially UTE2, are sensitive to off-resonance effects because of main magnetic field $\left(\mathrm{B}_{0}\right)$ inhomogeneity and susceptibility, causing inhomogeneity artifacts that make accurate image segmentation difficult (39). Thus, we generated a UTE MR-based attenuation map based on a level-set algorithm in which the intensity inhomogeneity correction was incorporated. These procedures were performed using in-house-developed code written in Matlab (R2014a; The MathWorks).

Level-Set Algorithm. Two-phase level-set segmentation based on the Chan and Vese multiphase model was applied to both the UTE1 and the UTE2, in which 2 level-set functions were evolved simultaneously (37). Local intensity clustering properties and region-based information were considered as proposed by $\mathrm{Li}$ et al. to unify the segmentation and inhomogeneity correction within a single evolving framework (38). Figure 1 shows the results of the level-set segmentation (the supplemental note provides detailed information on the level-set algorithm used in this study). The final evolved contours (red: level-set function $1=0$; blue: level-set function $2=0$ ) were overlaid on the MR images. The regions delimitated by the contours were represented in the binary images by assigning 1 to the inside of the contour and 0 to the outside. $\left(\mathrm{U}_{x} \mathrm{~L}_{y}\right.$ is the binary image from the $y$ th level-set function of the $x$ th UTE in Figure 1 . The symbol $\mathrm{C}$ labeled behind $y$ indicates that the binary image is generated with inhomogeneity correction.)

Generation of Attenuation Map. The procedure for generating the attenuation map is similar to Keereman's scheme (31). However, the accuracy of segmentation of each region was improved by the level-set method.

The soft-tissue map was obtained by applying a hole-filling operation to the $\mathrm{U}_{1} \mathrm{~L}_{1}$ that encloses almost all of the structures in the head. Air has a negligibly low signal in both UTE images. Thus, we obtained an air map by multiplying $\sim \mathrm{U}_{1} \mathrm{~L}_{1}$ and $\sim \mathrm{U}_{2} \mathrm{~L}_{1}$ (Fig. 1). To generate the bone 


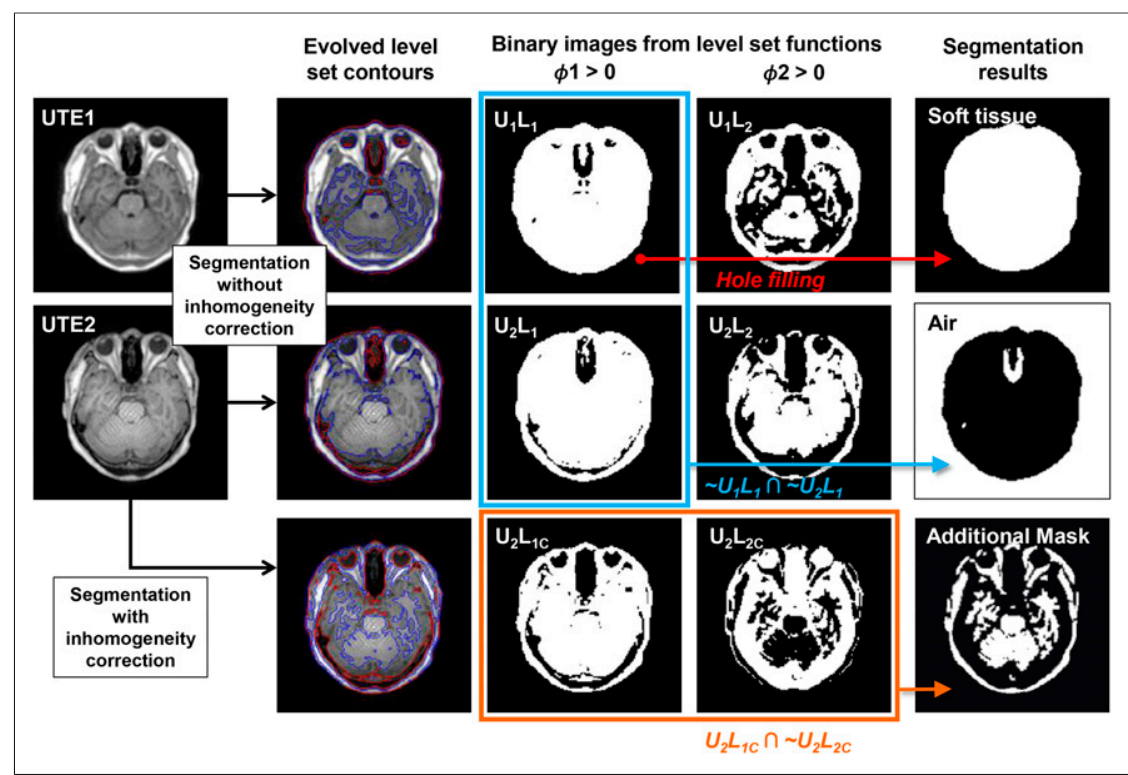

FIGURE 1. Generation of soft-tissue and air maps and additional mask using 2-phase level-set segmentation and morphologic and binary operations. Final evolved contours (red: level-set function $1=0$, blue: level-set function $2=0$ ) were overlaid on MR images. $U_{x} L_{y}$ was binary image from $x$ th level-set function of $y$ th UTE. Soft-tissue and air maps were generated by filling holes in $U_{1} L_{1}$ and multiplying 2 binary images $\left(\sim U_{1} L_{1}\right.$ and $\left.\sim U_{2} L_{1}\right)$. Additionally, mask for trimming bone map was generated from binary images of level-set functions obtained using level-set segmentation with inhomogeneity correction $\left(\mathrm{U}_{2} \mathrm{~L}_{1 \mathrm{C}} \cap \sim \mathrm{U}_{2} \mathrm{~L}_{2} \mathrm{C}\right)$.

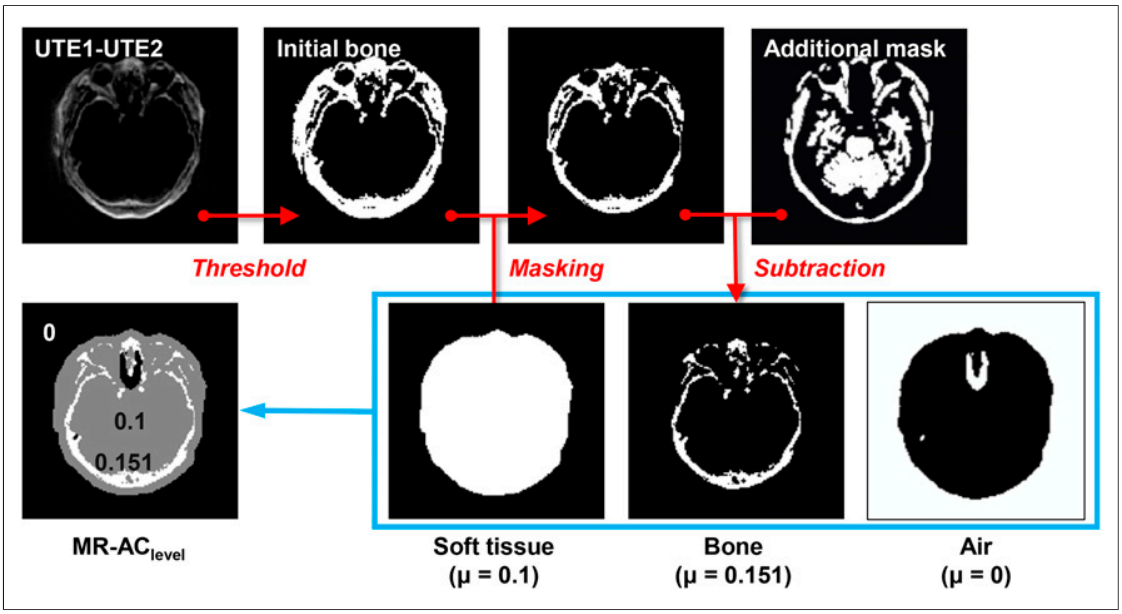

FIGURE 2. Generation of bone map and final level-set-based attenuation map (MR-AC level). Initial bone map generated by applying a threshold to difference image between UTE1 and UTE2 was further trimmed to yield final bone map by masking it with soft-tissue map and additional mask. MR-AC $\mathrm{Clel}_{\text {lel }}$ map was then generated by assigning attenuation coefficients to soft-tissue, bone, and air maps and combining them.

map, we started from the initial bone map generated by applying a threshold to the difference image (dUTE) between UTE1 and UTE2 (Fig. 2). The threshold was empirically determined and $50 \%$ of the mean intensity of dUTE pixels greater than 10 . This initial bone map was then masked by the morphologically eroded soft-tissue map to correct for the misclassified voxels around the outer boundary of the skull with air. To further trim out the remaining misclassified soft tissue as bone in the dUTE image, we applied an additional mask generated by multiplying $\mathrm{U}_{2} \mathrm{~L}_{1 \mathrm{C}}$ and $\sim \mathrm{U}_{2} \mathrm{~L}_{2 \mathrm{C}}$ (Figs. 1 and 2).

Finally, we added the bone segment to the initial attenuation map, and assigned the attenuation coefficients for soft tissue and bone
(0.1 and $\left.0.151 \mathrm{~cm}^{-1}\right)$. The computing time for generation of the attenuation map using the level-set method was approximately $5 \mathrm{~min}$ when this method was implemented using Matlab code (version R2014a) and executed on a personal computer with Intel Core i52500 Processor $(3.3 \mathrm{GHz})$.

\section{Image Processing and \\ Reconstruction}

Reconstructed PET images were generated from emission data in the PET/CT studies using 3 different attenuation maps. The first one was the MR-based attenuation map that is offered by the Biograph mMR software (MR$\mathrm{AC}_{\mathrm{mMR}}$ map). The second one was the MRbased attenuation map generated using the proposed multiphase level-set method (MR$\mathrm{AC}_{\text {level }}$ map). The last one was the CT-based attenuation map conventionally used in PET/CT studies, which was converted from the CT images to 511-keV attenuation coefficients using a bilinear transformation (CT-AC map).

For each participant, 2 MR-based attenuation maps were coregistered and resliced to the CT-AC map using the statistical parametric mapping (SPM8; University of College London) software through the coregistration of T1 3-dimensional MRI to CT. The $\mathrm{PET} / \mathrm{CT}$ head holder was visible in the CT-AC map, whereas the UTE images were without the head holder. Therefore, the head holder shown in the CT image was extracted using a region-growing segmentation algorithm and added to the MR-based attenuation maps to allow a fair comparison. All PET images were reconstructed using the ordinary Poisson ordered-subset expectation maximization (subsets, 14; iterations, 3) algorithm through e7tool from Siemens Healthcare. After reconstruction, all PET data were spatially normalized to the SPM standard MRI T1 template to eliminate intersubject anatomic variability. The overall image processing steps are summarized in Supplemental Figure 2.

\section{Image Analysis}

The quantitative accuracies of the 2 MR$\mathrm{AC}$ methods relative to CT-AC were compared using the similarity measurements of attenuation maps and absolute and relative differences between PET images.

The accuracy of the attenuation maps were evaluated using Dice similarity coefficients (40-42) that measure the overlap of the segmented bone and air regions of MR-AC maps with respect to those of CT-AC maps ( 1 for perfect overlap and 0 for no overlapping) according to the following equation:

$$
\mathrm{D}=\frac{2 \times N_{M R-A C} \cap C T-A C}{N_{M R-A C}+N_{C T-A C}}
$$

where $N$ is the number of bone (or air) voxels in each image. In the CTAC map, the voxels with $\mu$ greater than 0.1134 (=300 Hounsfield 


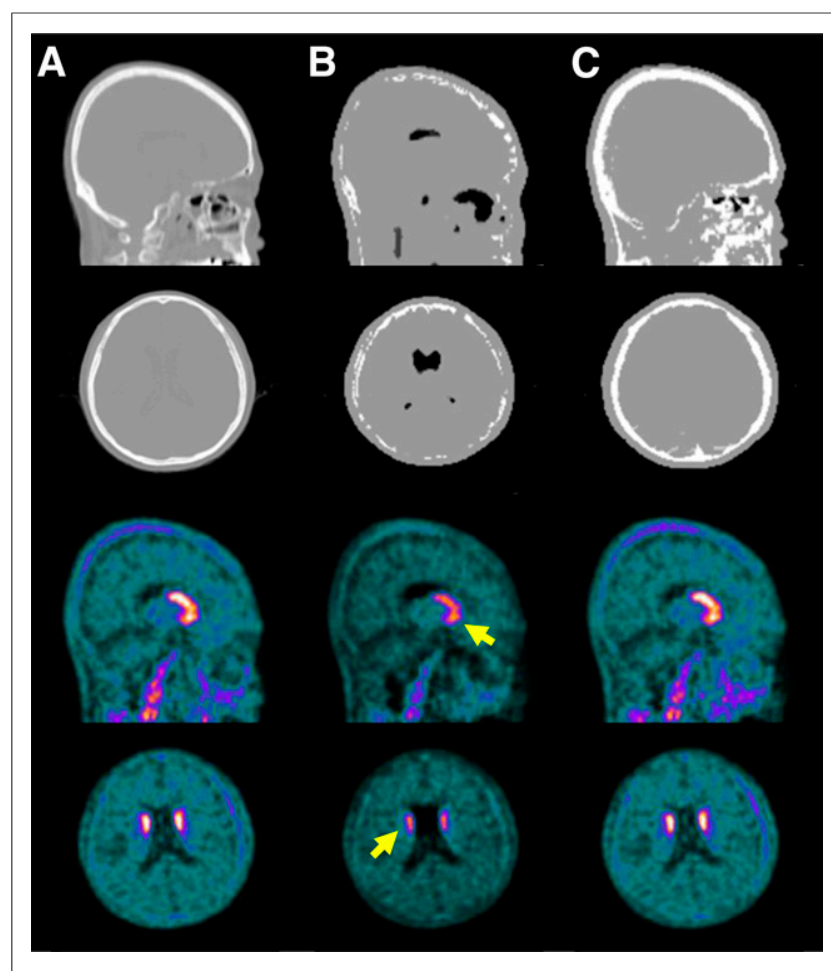

FIGURE 3. Attenuation maps and ${ }^{18} \mathrm{~F}-\mathrm{FP}-\mathrm{CIT}$ PET images corrected using them. (A) CT. (B) MR-AC ${ }_{m M R}$ : MR-based attenuation map generated using mMR software version VB18P. (C) MR-AC level: MR-based attenuation map generated using level-set method.

units) were classified into bone and the voxels with $\mu$ less than 0.0475 $(=-500$ Hounsfield units $)$ were air $(32,41)$. The Dice coefficients were calculated for the entire head and for the cranial region, separately (40).

We measured the PET activity concentration in 5 regions of interest (ROIs) (caudate nucleus, putamen, thalamus, occipital, and cerebellum) for ${ }^{18} \mathrm{~F}$-FP-CIT studies and 10 ROIs (frontal, temporal, parietal, occipital, insula, striatum, precuneus, amygdala/hippocampus, thalamus, and cerebellum) for ${ }^{18} \mathrm{~F}-\mathrm{FDG}$ studies using an automatic ROI-delineation method with statistical probabilistic anatomic maps (43). The mean SUV of each ROI and its ratio to the cerebellum (SUVr) were calculated. The relative ratio of specific binding (binding ratio, $\left[\mathrm{C}_{\text {specific }}-\mathrm{C}_{\text {nonspecific }}\right] / \mathrm{C}_{\text {nonspecific }}$ ) was also calculated to assess the ${ }^{18} \mathrm{~F}$-FP-CIT binding in the caudate nucleus and putamen $\left(\mathrm{C}_{\text {specific }}\right.$ and $\mathrm{C}_{\text {nonspecific }}$ : activity concentrations in specific and nonspecific [cerebellum] binding regions, respectively).

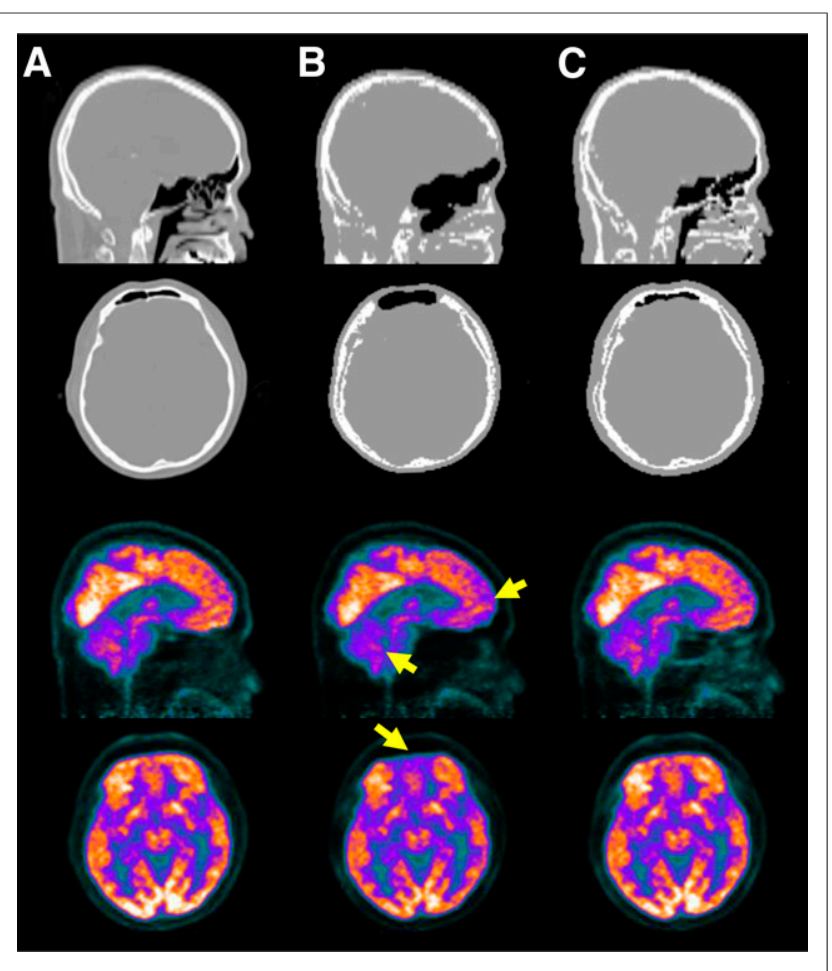

FIGURE 4. Attenuation maps and ${ }^{18} \mathrm{~F}-\mathrm{FDG}$ PET images corrected using them. (A) CT. (B) MR-AC ${ }_{m M R}$ using $m M R$ software version VB20P. (C) MR-AC level.

For the voxelwise comparison, all the PET images were spatially normalized as shown in Supplemental Figure 2, mean PET images of MR-AC and CT-AC were generated, and their absolute and relative difference maps were generated.

\section{RESULTS}

The results of CT-AC, MR-AC $\mathrm{mMR}_{\mathrm{m}}$, and $\mathrm{MR}-\mathrm{AC}_{\text {level }}$ applied to the same emission data acquired using PET/CT machines are compared in Figures 3 and 4 (Fig. 3 for ${ }^{18}$ F-FP-CIT PET with VB18P mMR software, Fig. 4 for ${ }^{18}$ F-FDG PET and VB20P). The MR-AC $\mathrm{mMR}_{\mathrm{mM}}$ map gave larger air cavities than $\mathrm{CT}$ regardless of the version of $\mathrm{mMR}$ software. The bone tissue in the $\mathrm{MR}-\mathrm{AC}_{\mathrm{mMR}}$ map was underestimated in VB18P (Fig. 3B) and overestimated in VB20P (Fig. 4B). On the contrary, MR-AC level maps (Figs. 3C

TABLE 1

Dice Similarity Coefficients for Whole Head and Cranial Bone (Mean \pm SD)

\begin{tabular}{|c|c|c|c|c|}
\hline \multirow[b]{2}{*}{ Method } & \multicolumn{2}{|c|}{ Whole head } & \multicolumn{2}{|c|}{ Cranial region } \\
\hline & $D_{\text {bone }}$ & $\mathrm{D}_{\text {air }}$ & $D_{\text {bone }}$ & $\mathrm{D}_{\text {air }}$ \\
\hline \multicolumn{5}{|c|}{${ }^{18} \mathrm{~F}-\mathrm{FP}-\mathrm{ClT}$ study $(n=20)$} \\
\hline MR-AC ${ }_{\mathrm{mMR}}(\mathrm{VP18P})$ & $0.28 \pm 0.09$ & $0.45 \pm 0.10$ & $0.31 \pm 0.11$ & $0.42 \pm 0.10$ \\
\hline MR-AC ${ }_{\text {level }}$ & $0.60 \pm 0.06$ & $0.54 \pm 0.09$ & $0.71 \pm 0.06$ & $0.59 \pm 0.08$ \\
\hline \multicolumn{5}{|l|}{${ }^{18} \mathrm{~F}-\mathrm{FDG}$ study $(n=10)$} \\
\hline$M R-A C_{m M R}(V P 20 P)$ & $0.72 \pm 0.04$ & $0.60 \pm 0.06$ & $0.74 \pm 0.04$ & $0.59 \pm 0.09$ \\
\hline MR-AC ${ }_{\text {level }}$ & $0.79 \pm 0.02$ & $0.61 \pm 0.07$ & $0.83 \pm 0.02$ & $0.62 \pm 0.10$ \\
\hline
\end{tabular}

Data are mean \pm SD. 


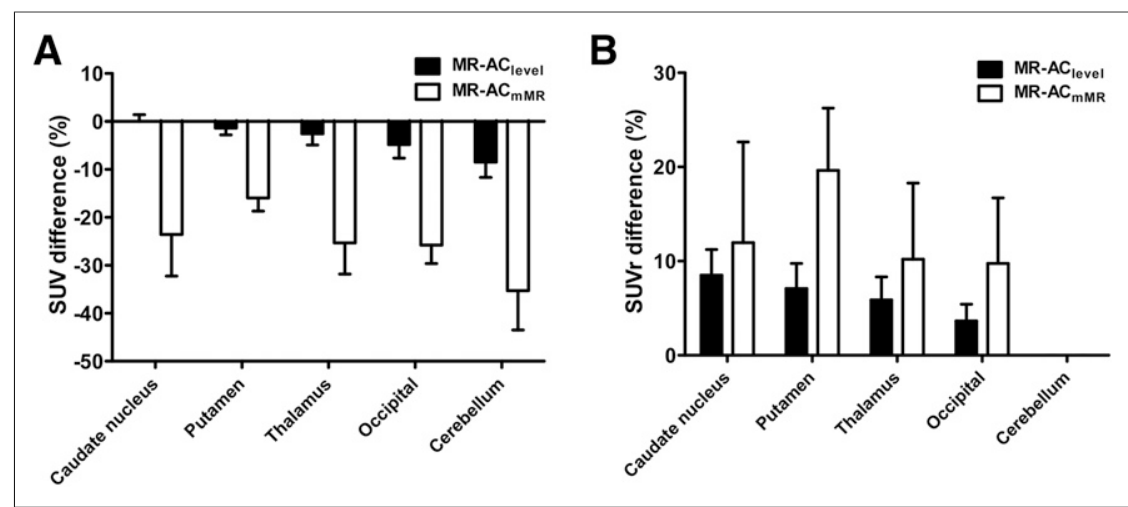

FIGURE 5. Percentage difference of SUV (A) and SUVr (B) from CT-AC in ${ }^{18} \mathrm{~F}-\mathrm{FP}-\mathrm{CIT}$ PET. that there was a remarkable difference in almost every brain region in the voxelwise comparison between MR-AC $\mathrm{mMR}_{\mathrm{m}}$ and $\mathrm{CT}$ AC. On the contrary, the difference between MR-AC $\mathrm{A}_{\text {level }}$ and CT-AC was limited to the brain cortex. In both methods, the outer boundary of brain cortex, which is vulnerable to the brain size mismatch and registration error between CT and MRI and errors in skull segmentation, showed the largest differences.

\section{DISCUSSION}

In this study, we developed a new UTE MR-AC map based on a unified multiphase level-set segmentation and inhomogeneity and 4C) showed more similar properties with CT (Figs. 3A and 4A) in the size and shape of the air cavities and bones. Although the striatal and cerebellar activity in ${ }^{18} \mathrm{~F}-\mathrm{FP}-\mathrm{CIT}$ PET and frontal activity in ${ }^{18} \mathrm{~F}$-FDG PET were remarkably underestimated in MR$\mathrm{AC}_{\mathrm{mMR}}$ relative to CT-AC (Figs. $3 \mathrm{~B}$ and $4 \mathrm{~B}$ ), MR-AC $\mathrm{Clvel}_{\text {lel }}$ did not show this discrepancy from CT-AC (Figs. 3C and 4C).

The Dice similarity coefficients between MR-AC and CT-AC maps are summarized in Table 1 . The mean Dice coefficients for

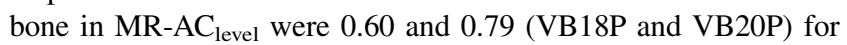
the whole head and 0.71 and 0.83 for the cranial region only, and all of them were higher than those in MR- $\mathrm{AC}_{\mathrm{mMR}}$. There was the same trend for air regions.

The superiority of MR-AC $\mathrm{C}_{\text {level }}$ to $\mathrm{MR}-\mathrm{AC}_{\mathrm{mMR}}$ was confirmed in the ROI- and voxel-based quantitative comparisons. Figures 5 and 6 show the results of ROI-based analysis on ${ }^{18} \mathrm{~F}$-FP-CIT and ${ }^{18} \mathrm{~F}-$ FDG PET data, respectively. In ${ }^{18}$ F-FP-CIT studies with MR$\mathrm{AC}_{\mathrm{mMR}}$, the percentage difference of SUV from CT-AC was greater than $-20 \%$ in most ROIs (Fig. 5A). The percentage difference was most remarkable in the cerebellum, leading to the overestimation of SUVr, which was highest in the putamen (Fig. 5B). Conversely, the percentage difference of ${ }^{18} \mathrm{~F}$-FP-CIT PET with MR-AC $C_{\text {level }}$ from CT-AC was smaller than $10 \%$ in both SUV and SUVr. The binding ratio values offered by the MR-AC methods were linearly correlated with those by CT-AC; nevertheless, the MR-AC $\mathrm{C}_{\text {level }}$ (putamen: $y=1.04 x \pm 0.016$; caudate: $y=1.04 x \pm$

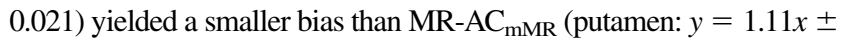
0.038; caudate: $y=1.14 x \pm 0.073$ ) (Supplemental Figs. 3-6).

The ${ }^{18} \mathrm{~F}-\mathrm{FDG}$ PET tests showed a trend similar to the ${ }^{18} \mathrm{~F}-\mathrm{FP}-$ CIT PET tests, whereas the percentage differences in SUV and SUVr between MR-AC and CT-AC were roughly half of those in ${ }^{18}$ F-FP-CIT PET (Fig. 6; Supplemental Figs. 7-9). Figure 7 shows correction method and demonstrated the superior performance of this method over the currently used MR-AC map in an mMR PET/ MRI scanner. The remarkable improvements in the segmentation of air cavities and bone and the quantitative accuracy of PET measurement using the level-set method were shown in both the ${ }^{18} \mathrm{~F}-\mathrm{FP}-\mathrm{CIT}$ PET data using VB18P mMR software and the ${ }^{18} \mathrm{~F}$ FDG PET data using VB20P.

The major upgrade of mMR software from VB18P to VB20P seems to be effective in the elimination of misclassification of cerebrospinal fluid in ventricles as air and the correction of bone underestimation shown in previous reports (33-35). The percentage error of MR-AC $\mathrm{mMR}_{\mathrm{m}}$ in $\mathrm{SUV}$ and $\mathrm{SUVr}$ quantification relative to CT-AC was reduced approximately by half, although we could not confirm this error reduction using the exact same dataset. However, the current VB20P version still yields air cavity and bone segmentation errors as shown in Figure 4B. However, the $\mathrm{MR}-\mathrm{AC}_{\text {level }}$ offered improved segmentation results, leading to the reduction of PET quantification error by a factor of approximately 3 as shown in Figure 5 (SUV error $<10 \%$ in MR- $\mathrm{AC}_{\text {level }}$ and $<30 \%$ in MR-AC $\mathrm{mMR}_{\mathrm{mM}}$ with VB18P, and $<5 \%$ in MR-AC $\mathrm{Alvel}_{\text {lel }}$ and $<15 \%$ in $\mathrm{MR}-\mathrm{AC}_{\mathrm{mMR}}$ with $\left.\mathrm{VB} 20 \mathrm{P}\right)$. The evaluation of attenuation maps using Dice coefficients confirmed the improvements in the MR-AC maps achieved by the level-set method (Table 1). For VB20P UTE datasets, $\mathrm{MR}-\mathrm{AC}_{\text {level }}$ yielded the Dice coefficient for bone of 0.83 in the cranial region whereas $\mathrm{MR}-\mathrm{AC}_{\mathrm{mMR}}$ offered 0.74 in this study and 0.65 in a previous study (40).

The results suggest that UTE MR-AC $\mathrm{A}_{\text {level }}$ provides more accurate PET quantification than Dixon-based AC methods that yielded around 10\%-20\% errors in the study by Dickson et al. (33) and $5 \%-15 \%$ in the study by Izquierdo-Garcia et al. (17) depending on brain regions (larger error in cortical regions). Recent advanced template-based approaches (17) and new approaches with $R^{2 *}$ to Hounsfield unit conversion (41) and zero-echo time (42) show results similar to our approach or great potential for further improvement of MR-AC. The combination of our approach with those methods would be the interesting next step that we can take to improve the MR-AC in brain and potentially in whole-body PET/MRI studies.

The advanced results using the level-set method can be attributed to the combined effects of various factors in this study. These factors include the inhomogeneity

FIGURE 6. Percentage difference of SUV (A) and SUVr (B) from CT-AC in ${ }^{18} \mathrm{~F}-\mathrm{FDG}$ PET. 


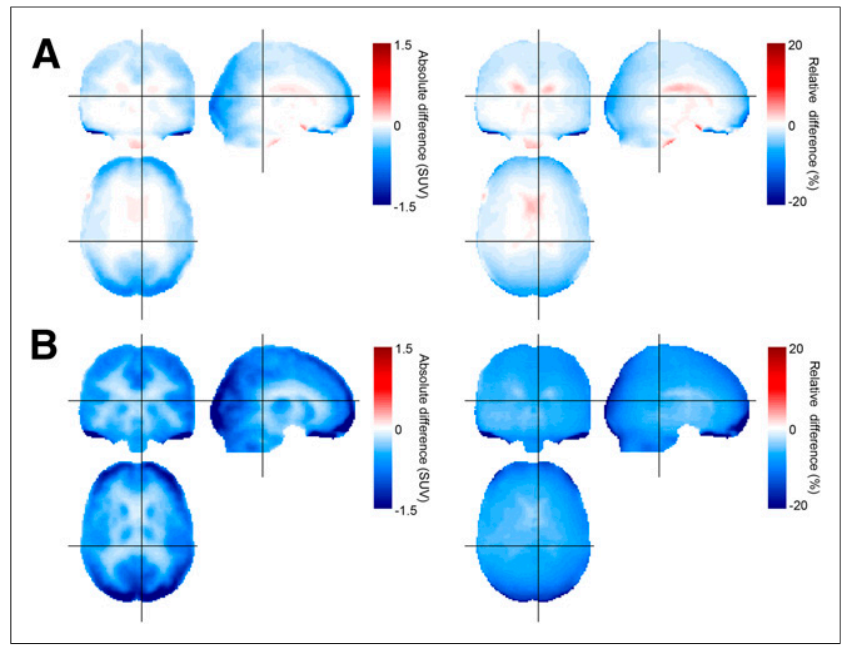

FIGURE 7. Absolute (left) and percentage (right) difference of SUV in ${ }^{18} \mathrm{~F}-\mathrm{FDG}$ PET images. (A) MR-AClevel with respect to CT-AC. (B) MR-AC $\mathrm{mMR}_{\mathrm{mM}}$ with respect to CT-AC.

correction of UTE images incorporated into the level-set segmentation, which led to the more reliable segmentation results. The assorted boundary information provided by the multiphase levelset segmentations applied to both the UTE images were useful for determining the complex boundaries among different segments and trimming the segmentation results through morphologic operations on the binary images.

Although MR-AC $\mathrm{Alvel}_{\text {lev }}$ yielded SUV quantification results almost equivalent to $\mathrm{CT}-\mathrm{AC}$ in most brain regions, the errors in the cerebellum and occipital cortex were larger than in other regions (Figs. $5 \mathrm{~A}$ and $6 \mathrm{~A}$ ). The errors in these most common reference regions in brain PET studies resulted in positive biases in binding ratios and SUVr estimations (Figs. 5B and 6B; Supplemental Figs. 5 and 6). It is most likely that the errors in these posterior and inferior brain regions are related to the misclassification of fat tissues in the neck as bone. This misclassification, also observed in Figures $3 \mathrm{C}$ and $4 \mathrm{C}$, is likely caused by the image intensity brightening at the periphery of UTE images mainly because of the inhomogeneous $B_{1}$ field associated with multichannel phased array coils $(39,44)$.

Thus, we expect to achieve more accurate UTE segmentation and MR-based attenuation correction through the further optimization of UTE sequences (i.e., the reduction of off-resonance effects, robustness enhancement of non-Cartesian data acquisition, and saturation of fat tissues) (45).

\section{CONCLUSION}

We have developed a UTE MR-AC method using level-set segmentation with inhomogeneity correction for brain PET/MRI studies and demonstrated the feasibility of this method in brain PET/MRI studies with ${ }^{18}$ F-FP-CIT and ${ }^{18}$ F-FDG. The MR-based attenuation maps generated using level-set segmentation and PET images corrected for attenuation and scatter were superior to those offered by the manufacturer of the PET/MRI system in terms of the similarity to the CT-AC. This method will be useful for improving the quantitative accuracy of brain PET in PET/MRI studies.

\section{DISCLOSURE}

The costs of publication of this article were defrayed in part by the payment of page charges. Therefore, and solely to indicate this fact, this article is hereby marked "advertisement" in accordance with 18 USC section 1734. This work was supported by grants from the Ministry of Trade, Industry \& Energy (10030030); the National Research Foundation of Korea (NRF-2014M3C7034000); and the Korea Health Industry Development Institute (HI13C01630200) and an Intramural Research Grant of Seoul National University (grant 0420130670, 2013-1290). No other potential conflict of interest relevant to this article was reported.

\section{REFERENCES}

1. Silverman DH. Brain ${ }^{18} \mathrm{~F}-\mathrm{FDG}$ PET in the diagnosis of neurodegenerative dementias: comparison with perfusion SPECT and with clinical evaluations lacking nuclear imaging. J Nucl Med. 2004;45:594-607.

2. Kazumata K, Dhawan V, Chaly T, et al. Dopamine transporter imaging with fluorine-18-FPCIT and PET. J Nucl Med. 1998;39:1521-1530.

3. Biswal B, Yetkin FZ, Haughton VM, Hyde JS. Functional connectivity in the motor cortex of resting human brain using echo-planar MRI. Magn Reson Med. 1995;34:537-541.

4. Frisoni GB, Fox NC, Jack CR Jr, Scheltens P, Thompson PM. The clinical use of structural MRI in Alzheimer disease. Nat Rev Neurol. 2010;6:67-77.

5. Catana C, Drzezga A, Heiss WD, et al. PET/MRI for neurologic applications. J Nucl Med. 2012;53:1916-1925.

6. Drzezga A, Barthel H, Minoshima M, et al. Potential clinical applications of PET/MR imaging in neurodegenerative disease. J Nucl Med. 2014;55:47S-55S.

7. Pichler BJ, Judenhofer MS, Catana C, et al. Performance test of an LSO-APD detector in a 7-T MRI scanner for simultaneous PET/MRI. J Nucl Med. 2006;47:639-647.

8. Catana C, Wu Y, Judenhofer MS, et al. Simultaneous acquisition of multislice PET and MR images: initial results with a MR-compatible PET scanner. J Nucl Med. 2006;47:1968-1976.

9. Schlemmer HP, Pichler B, Schm M, et al. Simultaneous MR/PET imaging of the human brain: feasibility study. Radiology. 2008;248:1028-1035.

10. Yoon HS, Ko GB, Kwon SI, et al. Initial results of simultaneous PET/MRI experiments with an MRI-compatible silicon photomultiplier PET scanner. J Nucl Med. 2012;53:608-614.

11. Yamamoto S, Watabe T, Watabe H, et al. Simultaneous imaging using Si-PMbased PET and MRI for development of an integrated PET/MRI system. Phys Med Biol. 2012;57:N1-N13.

12. Wehrl HF, Hossain M, Lankes K, et al. Simultaneous PET-MRI reveals brain function in activated and resting state on metabolic, hemodynamic and multiple temporal scales. Nat Med. 2013;19:1184-1189.

13. Sander CY, Hooker JM, Catana C, et al. Neurovascular coupling to D2/D3 dopamine receptor occupancy using simultaneous PET/functional MRI. Proc Natl Acad Sci USA. 2013;110:11169-11174.

14. Lee JS, Kim JH. Recent advances in hybrid molecular imaging systems. Semin Musculoskelet Radiol. 2014;18:103-122.

15. Keereman V, Mollet P, Berker Y, et al. Challenges and current methods for attenuation correction in PET/MR. MAGMA. 2013;26:81-98.

16. Wagenknecht G, Kaiser H-J, Mottaghy FM, et al. MRI for attenuation correction in PET: methods and challenges. MAGMA. 2013;26:99-113.

17. Izquierdo-Garcia D, Hansen AE, Förster S, et al. An SPM8-based approach for attenuation correction combining segmentation and nonrigid template formation: application to simultaneous PET/MR brain imaging. J Nucl Med. 2014;55:1825-1830.

18. Yoo HJ, Lee JS, Jee JM. Integrated whole body PET/MRI: where are we? Korean J Radiol. 2015;16:32-49.

19. Montandon M-L, Zaidi H. Atlas-guided non-uniform attenuation correction in cerebral 3D PET imaging. Neuroimage. 2005;25:278-286.

20. Kops ER, Herzog H. Alternative methods for attenuation correction for PET images in MR-PET scanners. Nucl Sci Symp Conf Rec. 2007;6:4327-4330.

21. Malone IB, Ansorge RE, Williams GB, et al. Attenuation correction methods suitable for brain imaging with a PET/MRI scanner: a comparison of tissue atlas and template attenuation map approaches. J Nucl Med. 2011;52:1142-1149.

22. Hofmann M, Bezrukov I, Mantlik F, et al. MRI-based attenuation correction for whole-body PET/MRI: quantitative evaluation of segmentation- and Atlas-based methods. J Nucl Med. 2011;52:1392-1399.

23. Martinez-Möller A, Souvatzoglou M, Delso G, et al. Tissue classification as a potential approach for attenuation correction in whole-body PET/MRI: evaluation with PET/CT data. J Nucl Med. 2009;50:520-526.

24. Keereman V, Holen RV, Mollet P, et al. The effect of errors in segmented attenuation maps on PET quantification. Med Phys. 2011;38:6010-6019.

25. Berker Y, Franke J, Salomon A, et al. MRI-based attenuation correction for hybrid PET/MRI systems: a 4-class tissue segmentation technique using a 
combined ultrashort-echo-time/Dixon MRI sequence. J Nucl Med. 2012;53: 796-804.

26. Samarin A, Burger C, Wollenweber SD, et al. PET/MR imaging of bone lesionsimplications for PET quantification from imperfect attenuation correction. Eur J Nucl Med Mol Imaging. 2012;39:1154-1160.

27. Kim JH, Lee JS, Song IC, et al. Comparison of segmentation-based attenuation correction methods for PET/MRI: evaluation of bone and liver standardized uptake value with oncologic PET/CT data. J Nucl Med. 2012;53:1878-1882.

28. Nuyts J, Dupont P, Stroobants S, et al. Simultaneous maximum a posteriori reconstruction of attenuation and activity distributions from emission sonograms. IEEE Trans Med Imaging. 1999;18:393-403.

29. Salomon A, Goedicke A, Schweizer B, et al. Simultaneous reconstruction of activity and attenuation for PET/MR. IEEE Trans Med Imaging. 2011;30:804813.

30. Rezaei A, Defrise M, Bal G, et al. Simultaneous reconstruction of activity and attenuation in time-of-flight PET. IEEE Trans Med Imaging. 2012;31:22242233.

31. Keereman V, Fierens Y, Broux T, et al. MRI-based attenuation correction for PET/MRI using ultrashort echo time sequences. J Nucl Med. 2010;51:812-818.

32. Catana C, van der Kouwe A. Benner, et al. T Toward implementing an MRIbased PET attenuation-correction method for neurologic studies on the MR-PET brain prototype. J Nucl Med. 2010;51:1431-1438.

33. Dickson JC, O'Meara C, Barnes A. A comparison of CT- and MR-based attenuation correction in neurological PET. Eur J Nucl Med Mol Imaging. 2014;41:1176-1189.

34. Cabello J, Lukas M, Förster S, et al. MR-based attenuation correction using ultrashort-echo-time pulse sequences in dementia patients. J Nucl Med. 2015;56:423-429.
35. Choi H, Cheon GJ, Kim H-J, et al. Segmentation-based MR attenuation correction including bones also affects quantitation in brain studies: an initial result of ${ }^{18} \mathrm{~F}-\mathrm{FP}$ CIT PET/MR for patients with parkinsonism. J Nucl Med. 2014;55:1617-1622.

36. Chan TF, Vese LA. Active contours without edges. IEEE Trans Image Process. 2001;10:266-277.

37. Vese LA, Chan TF. A multiphase level set framework for image segmentation using the Mumford and Shah model. Int J Comput Vis. 2002;53:271-293.

38. Li C, Huang R, Ding Z, et al. A level set method for image segmentation in the presence of intensity inhomogeneities with application to MRI. IEEE Trans Image Process. 2011;20:2007-2016.

39. Robson MD, Gatehouse PD, Bydder M, et al. Magnetic resonance: an introduction to ultrashort TE (UTE) imaging. J Comput Assist Tomogr. 2003;27:825-846.

40. Aasheim LB, Karlberg A, Goa PE, et al. PET/MR brain imaging: evaluation of clinical UTE-based attenuation correction. Eur J Nucl Med Mol Imaging. 2015;42:1439-1446.

41. Juttukonda MR, Mersereau BG, Chen Y, et al. MR-based attenuation correction for PET/MRI neurological studies with continuous-valued attenuation coefficients for bone through a conversion from R2* to CT-Hounsfield units. Neuroimage. 2015;112:160-168.

42. Delso G, Wiesinger F, Sacolick LI, et al. Clinical evaluation of zero-echo-time MR imaging for the segmentation of the skull. J Nucl Med. 2015;56:417-422.

43. Lee JS, Lee DS. Analysis of functional brain images using population-based probabilistic atlas. Curr Med Imaging Rev. 2005;1:81-87.

44. Pilkinton DT, Elliott MA, Magland J. Sodium MRI multi-echo sequence for simultaneous ultra-short echo imaging and T2L* mapping at 7T with a 12 channel phased-array coil [abstract]. Proc Intl Soc Mag Reson Med. 2010;18:1002.

45. Aitken AP, Giese D, Tsoumpas C, et al. Improved UTE-based attenuation correction for cranial PET-MR using dynamic magnetic field monitoring. Med Phys. 2014;41:012302. 